\title{
Response and survival of breast cancer intrinsic subtypes following multi-agent neoadjuvant chemotherapy
}

\author{
Aleix Prat ${ }^{1,2,3^{*}}$ (D), Cheng Fan ${ }^{4}$, Aranzazu Fernández ${ }^{2,3}$, Katherine A. Hoadley ${ }^{4}$, Rossella Martinello ${ }^{2,3}$, Maria Vidal ${ }^{1}$, \\ Margarita Viladot ${ }^{2,3}$, Estela Pineda ${ }^{2,3}$, Ana Arance ${ }^{2,3}$, Montserrat Muñoz ${ }^{2,3}$, Laia Paré ${ }^{2,3}$, Maggie C. U. Cheang ${ }^{5}$, \\ Barbara Adamo ${ }^{2,3}$ and Charles M. Perou ${ }^{4,6,7}$
}

\begin{abstract}
Background: Predicting treatment benefit and/or outcome before any therapeutic intervention has taken place would be clinically very useful. Herein, we evaluate the ability of the intrinsic subtypes and the risk of relapse score at diagnosis to predict survival and response following neoadjuvant chemotherapy. In addition, we evaluated the ability of the Claudin-low and 7-TNBCtype classifications to predict response within triple-negative breast cancer (TNBC).

Methods: Gene expression and clinical-pathological data were evaluated in a combined dataset of 957 breast cancer patients, including 350 with TNBC, treated with sequential anthracycline and anti-microtubule-based neoadjuvant regimens. Intrinsic subtype, risk of relapse score based on subtype and proliferation (ROR-P), the Claudin-low subtype and the 7-TNBCtype subtype classification were evaluated. Logistic regression models for pathological complete response ( $\mathrm{pCR}$ ) and Cox models for distant relapse-free survival (DRFS) were used.

Results: Basal-like, Luminal A, Luminal B, and HER2-enriched subtypes represented 32.7 \%, 30.6 \%, 18.2 \%, and $10.3 \%$ of cases, respectively. Intrinsic subtype was independently associated with pCR in all patients, in hormone receptor-positive/ HER2-negative disease, in HER2-positive disease, and in TNBC. The pCR rate of Basal-like disease was $>35 \%$ across all clinical cohorts. Neither the Claudin-low nor the 7-TNBCtype subtype classifications predicted pCR within TNBCs after accounting for intrinsic subtype. Finally, intrinsic subtype and ROR-P provided independent prognostic information beyond clinicopathological variables and type of pathological response. A 5 -year DRFS of $97.5 \%$ (92.8-100.0 \%) was observed in these neoadjuvant-treated and clinically node-negative patients predicted to be low risk by ROR-P (i.e. $57.4 \%$ of Luminal A tumors with clinically node-negative disease).

Conclusions: Intrinsic subtyping at diagnosis provides prognostic and predictive information for patients receiving neoadjuvant chemotherapy. Although we could not exclude a survival benefit of neoadjuvant chemotherapy in patients with early breast cancer with clinically node-negative and ROR-low disease at diagnosis, the absolute benefit of cytotoxic therapy in this group might be rather small (if any).
\end{abstract}

Keywords: Biomarker, Breast cancer, Gene expression, Intrinsic subtypes, Triple-negative

\footnotetext{
* Correspondence: alprat@clinic.ub.es

'Translational Genomics Group, Vall d'Hebron Institute of Oncology (VHIO), Barcelona, Spain

${ }^{2}$ Translational Genomics and Targeted Therapeutics in Solid Tumors, August

Pi i Sunyer Biomedical Research Institute (IDIBAPS), Rosselló, 149, 08036

Barcelona, Spain

Full list of author information is available at the end of the article
} 


\section{Background}

During the last decade, it has become apparent that gene expression-based data in breast cancer can provide useful biological, prognostic, and predictive information [1, 2]. For example, the main intrinsic molecular subtypes of breast cancer (Luminal A, Luminal B, HER2enriched, and Basal-like) are biologically and prognostically relevant [3-6] and have been associated with anthracycline and tamoxifen benefit in the adjuvant setting [7-9]. Importantly, the intrinsic subtypes are not fully recapitulated by the combined determination of pathology-based biomarkers such as estrogen receptor (ER), progesterone receptor (PR), Ki67, and HER2 [1, 3, $4,9-12]$, all of which are currently being used in the clinical setting. Thus, from a clinical perspective, there is a need to understand the value of identifying the intrinsic subtypes, as well as other gene expression-based classifications, beyond clinicopathological variables.

We have previously shown that all the intrinsic subtypes can be identified within various clinically-defined groups, albeit with different proportions [9, 11, 13, 14]. For example, although the Basal-like subtype predominates within triple-negative breast cancer (TNBC), all the intrinsic subtypes can be identified in TNBC, and identification of the 'Basal-like versus not' classification within TNBC might be clinically relevant $[15,16]$. Beyond the main subtypes of breast cancer, we have also reported the Claudin-low subtype characterized by the low to absent expression of luminal differentiation markers, and by the high enrichment for epithelial-to-mesenchymal transition markers, immune response genes, and cancer stem cell-like features [4]. In a previous report, Claudin-low tumors showed an intermediate pathological complete response $(\mathrm{pCR})$ rate compared to Basal-like tumors in a cohort of 133 patients with TNBC and non-TNBC tumors treated with anthracycline/taxane-based chemotherapy [4].

Recently, Lehmann et al. [17] reported the identification of seven different potential molecular subtypes of TNBC (Basal 1 (BL1), Basal 2 (BL2), Immunomodulatory, Luminal androgen receptor (LAR), Mesenchymal, Mesenchymal stem cell (MSL), and unstable UNS). This seven-subtype classification of TNBC was found to be associated with pCR in an independent cohort of 130 TNBC patients treated with anthracycline/taxane-based chemotherapy [18]. Among the different subtypes, BL2 and LAR subtypes showed the lowest pCR rates, and BL1 showed the highest $\mathrm{pCR}$ rates, compared to the other subtypes [18].

In this study, we evaluated the ability of the common PAM50 intrinsic subtypes, and the risk of relapse score based on subtype and proliferation (ROR-P), to predict response and survival outcomes beyond standard clinicalpathological variables following neoadjuvant multi-agent chemotherapy. In addition, we evaluated the ability of the
Claudin-low [4] and the seven TNBC subtype classifications [17] to predict pCR within TNBC. Finally, we trained and tested gene expression-based models predictive of pCR in all patients, in patients with Basal-like disease, and in patients with Luminal disease, to identify some of the driving biological features behind response within these groups.

\section{Methods}

\section{Patients, samples and clinical data}

Four clinically annotated microarray-based breast cancer datasets were evaluated from the public domain (GSE25066 [19], GSE32646 [20], GSE41998 [21], and GSE22226 [22]). All patients received sequential anthracycline and taxane/exabepilone-based neoadjuvant regimens. Patients that received trastuzumab were excluded. All gene expression microarray-based analyses were performed in pre-treatment tumor samples. The total number of patients included in this analysis was 957 (Additional file 1: Figure S1). Ethical approval and informed consent were not required for this study.

The Hatzis et al. [19] dataset includes 508 patients treated with sequential anthracycline and taxane-based chemotherapy in various research protocols: LAB99-402, USO-02-103, 2003-0321, and I-SPY-1. A total of 508 patients from the Hatzis et al. [19] dataset have follow-up data. Patients with any nuclear immunostaining of ER in the tumor cells were considered eligible for adjuvant endocrine therapy. In Horak et al. [21], 279 patients were randomized to four cycles of doxorubicin/cyclophosphamide followed by 1:1 randomization to either ixabepilone $40 \mathrm{mg} / \mathrm{m}^{2}$ every 3 weeks for four cycles or weekly paclitaxel $80 \mathrm{mg} / \mathrm{m}^{2}$ for 12 weeks, followed by either weekly paclitaxel or exabepilone for 3 months. In Miyake et al. [20], 115 patients received paclitaxel $\left(80 \mathrm{mg} / \mathrm{m}^{2}\right)$ weekly for 12 cycles followed by 5 -FU $\left(500 \mathrm{mg} / \mathrm{m}^{2}\right)$, epirubicin $\left(75 \mathrm{mg} / \mathrm{m}^{2}\right)$ and cyclophosphamide $\left(500 \mathrm{mg} / \mathrm{m}^{2}\right)$ every 3 weeks for four cycles. Finally, Essermann et al. [22] included 149 patients treated in the ISPY-1 clinical trial with doxorubicin/ cyclophosphamide followed by paclitaxel. In this dataset, we excluded 80 patients that were already included in Hatzis et al. [19], one patient that received doxorubicin/ cyclophosphamide-only, and 13 patients that received trastuzumab.

\section{Pathology-based subtype definitions}

We used the pathological ER, PR, and HER2 statuses of each tumor sample as provided in each dataset [19-22]. The following pathology-based subtype definitions were evaluated: hormone receptor $(\mathrm{HR})^{+} / \mathrm{HER} 2^{-}, \mathrm{HER}^{+}$, and TNBC. 
Pathological complete response ( $p C R)$ definition

pCR across all cohorts was defined as the percentage of patients with no histologic evidence of residual invasive carcinoma in the breast and axillary lymph nodes, regardless of the presence or absence of ductal carcinoma in situ.

\section{Identification of the intrinsic subtypes}

In each dataset, all tumors were assigned to an intrinsic molecular subtypes of breast cancer (Luminal A, Luminal B, HER2-enriched, Basal-like) and the Normal breast-like group using the PAM50 subtype predictor as previously described [4, 22-24]. For the ISPY-1 [22] and Miyake [20] cohorts, we used the previously reported subtype calls [22, 25]. In addition, we evaluated the previously reported ROR-P score [23]. To identify the Claudin-low subtype [4] in TNBC, we applied the nine cell-line Claudin-low predictor in each microarray dataset using all patients as previously described [4]. TNBCs that were identified as Claudin-low were considered Claudinlow regardless of the intrinsic subtype call.

\section{Identification of subtypes within TNBC}

To identify the seven TNBC subtypes described by Lehmann et al. [17], we first selected the TNBCs from each dataset. Secondly, we submitted the raw data of each individual dataset to the TNBCtype online predictor (http://cbc.mc.vanderbilt.edu/tnbc/) [26]. The TNBCtype tool first checks the levels of the ER gene (ESR1) across all TNBCs, and identifies those samples with a relative high ESR1 expression level. These ESRhigh TNBCs need to be removed from each dataset in order for the TNBCtype predictor algorithm to continue.

\section{Training and testing gene expression-based models}

We explored the ability of newly derived gene expressionbased models to predict pCR in three different cohorts: all patients, patients with Basal-like disease, and patients with Luminal disease (Luminal A and B combined). To build each model, we explored the expression of 378 different gene signatures (Additional file 2: Supplemental Data) and used Elastic Net building model by 10 cross-validations. To accomplish this, we used the MDACC-based cohort (GSE25066 [19]) as a training set where each model was derived in each cohort, and then tested this exact model in the same clinical cohorts on the other datasets (testing sets). To estimate the performance of each model, we used the area under the receiver operating characteristic (auROC) curves.

\section{Statistical analysis}

Biologic analysis of the gene list was performed with DAVID annotation tool (http://david.abcc.ncifcrf.gov/) [27]. Association between subtype and pCR was assessed by univariate and multivariable logistic regression analysis. Likelihood ratio tests were used to assess if a variable added predictive information to each model. To estimate the predictive performance of each variable, auROC curves were evaluated. Survival functions to distant relapse-free survival (DRFS) were from the KaplanMeier product-limit estimator with tests of differences by the log-rank test. Cox proportional hazard models adjusted for standard clinical-pathological variables were used to test the independent associations with survival of each variable. Reported $P$ values are two-sided.

\section{Results \\ Clinical-pathological characteristics of the combined cohort}

A total of 957 patients with breast cancer treated with sequential anthracycline and taxane/ixabepilone-based neoadjuvant regimens were included in the analysis (Table 1). All datasets included all clinicopathological variables, except for histological grade and nodal status in Horak et al. [19] and nodal status in ISPY-1 et al. [22] since these were not provided. The mean age was 50.0 years and most patients had tumors of less than $5 \mathrm{~cm}(61.3 \%$ T0-T2) and positive axillary nodal status by clinical assessment (69.7\%). Pathology-based subtype distribution was as follows: $494(52.7 \%) \mathrm{HR}^{+} / \mathrm{HER}^{-}, 93$ (9.9 \%) HER2 ${ }^{+}$, and 350 (37.4 \%) TNBCs.

\section{Intrinsic subtype and ROR-P associations with survival outcome}

A total of 508 patients from Hatzis et al. [19] had follow-up data (mean 2.98 years). In this dataset, both intrinsic subtype and ROR-P were found to be significantly associated with DRFS in univariate and multivariable analyses after adjustment for age, tumor size, nodal status, ER and PR status, HER2 status, histological grade, and tumor response ( $\mathrm{pCR}$ vs. residual disease) (Additional file 1: Table S1 and S2). Of note, a 5-year DRFS rate of $90.2 \%$ (95\% confidence interval (CI), 82.5-98.6\%) was observed in patients whose tumors were predicted to be low risk by ROR-P (Additional file 1: Figure S2A). This 5-year DRFS rate increased to $97.5 \%$ (95\% CI, 92.78-100.0 \%) in patients with ROR-P low disease that presented with clinically node-negative disease (Additional file 1: Figure S2B).

Next, we evaluated the survival outcomes based on the type of pathological response. Within patients that achieved a pCR, no variable was found to be significantly associated with DRFS in univariate analyses (Fig. 1a and b; Additional file 1: Tables S3 and S4). Within patients that did not achieve a pCR, both intrinsic subtype and ROR-P were found to be significantly associated with DRFS in univariate and multivariable analyses after adjustment for the other clinicopathological 
Table 1 Clinicopathological characteristics and subtype distribution of the combined cohort evaluated in this study

\begin{tabular}{|c|c|c|c|c|c|c|c|c|c|c|}
\hline & GSE41998 [21] & $\%$ & GSE25066 [19] & $\%$ & GSE32646 [20] & $\%$ & GSE22226 [22] & $\%$ & TOTAL & $\%$ \\
\hline $\mathrm{N}$ & 279 & - & 508 & - & 115 & - & 55 & - & 957 & - \\
\hline Age, years (mean) & 49 & - & 50 & - & 51 & - & 49 & - & 50 & - \\
\hline \multicolumn{11}{|l|}{ Tumor size } \\
\hline T0-T2 & 177 & $64.1 \%$ & 288 & $56.7 \%$ & 92 & $80 \%$ & 27 & $50.0 \%$ & 584 & $61.3 \%$ \\
\hline T3-T4 & 99 & $35.9 \%$ & 220 & $43.3 \%$ & 23 & $20 \%$ & 27 & $50.0 \%$ & 369 & $38.7 \%$ \\
\hline \multicolumn{11}{|l|}{ ER IHC status } \\
\hline Positive & 108 & $38.7 \%$ & 297 & $59.2 \%$ & 71 & $62 \%$ & 31 & $58.5 \%$ & 507 & $53.4 \%$ \\
\hline Negative & 171 & $61.3 \%$ & 205 & $40.8 \%$ & 44 & $38 \%$ & 22 & $41.5 \%$ & 442 & $46.6 \%$ \\
\hline \multicolumn{11}{|l|}{ PR IHC status } \\
\hline Positive & 99 & $35.6 \%$ & 243 & $48.5 \%$ & 45 & $39 \%$ & 21 & $39.6 \%$ & 408 & $43.1 \%$ \\
\hline Negative & 179 & $64.4 \%$ & 258 & $51.5 \%$ & 70 & $61 \%$ & 32 & $60.4 \%$ & 539 & $56.9 \%$ \\
\hline \multicolumn{11}{|c|}{ Triple-negative status } \\
\hline No & 139 & $49.8 \%$ & 330 & $65.0 \%$ & 89 & $77 \%$ & 47 & $88.7 \%$ & 605 & $63.4 \%$ \\
\hline Yes & 140 & $50.2 \%$ & 178 & $35.0 \%$ & 26 & $23 \%$ & 6 & $11.3 \%$ & 350 & $36.6 \%$ \\
\hline \multicolumn{11}{|c|}{ HER2 IHC/FISH status } \\
\hline Negative & 251 & $90.0 \%$ & 485 & $98.8 \%$ & 81 & $70 \%$ & 24 & $49.0 \%$ & 841 & $90.0 \%$ \\
\hline Positive & 28 & $10.0 \%$ & 6 & $1.2 \%$ & 34 & $30 \%$ & 25 & $51.0 \%$ & 93 & $10.0 \%$ \\
\hline \multicolumn{11}{|l|}{ Histological grade } \\
\hline 1 & - & - & 32 & $6.8 \%$ & 16 & $13.9 \%$ & 2 & $3.6 \%$ & 50 & $14.9 \%$ \\
\hline 2 & - & - & 180 & $38.2 \%$ & 78 & $67.8 \%$ & 27 & $49.1 \%$ & 285 & $48.2 \%$ \\
\hline 3 & - & - & 259 & $55.0 \%$ & 21 & $18.3 \%$ & 26 & $47.3 \%$ & 306 & $51.8 \%$ \\
\hline \multicolumn{11}{|l|}{ Nodal status } \\
\hline No & - & - & 157 & $31 \%$ & 32 & $28 \%$ & - & - & 189 & $30.3 \%$ \\
\hline N1-3 & - & - & 351 & $69 \%$ & 83 & $72 \%$ & - & - & 434 & $69.7 \%$ \\
\hline \multicolumn{11}{|l|}{ pCR rate } \\
\hline No & 184 & $72.7 \%$ & 389 & $79.7 \%$ & 88 & $77 \%$ & 37 & $68.5 \%$ & 698 & $76.7 \%$ \\
\hline Yes & 69 & $27.3 \%$ & 99 & $20.3 \%$ & 27 & $23 \%$ & 17 & $31.5 \%$ & 212 & $23.3 \%$ \\
\hline \multicolumn{11}{|l|}{ PAM50 } \\
\hline Luminal A & 91 & $32.6 \%$ & 155 & $30.5 \%$ & 30 & $26 \%$ & 17 & $30.9 \%$ & 293 & $30.6 \%$ \\
\hline Luminal B & 33 & $11.8 \%$ & 109 & $21.5 \%$ & 23 & $20 \%$ & 9 & $16.4 \%$ & 174 & $18.2 \%$ \\
\hline HER2-E & 23 & $8.2 \%$ & 40 & $7.9 \%$ & 24 & $21 \%$ & 12 & $21.8 \%$ & 99 & $10.3 \%$ \\
\hline Basal-like & 110 & $39.4 \%$ & 171 & $33.7 \%$ & 21 & $18 \%$ & 11 & $20.0 \%$ & 313 & $32.7 \%$ \\
\hline Normal-like & 22 & $7.9 \%$ & 33 & $6.5 \%$ & 17 & $15 \%$ & 6 & $10.9 \%$ & 78 & $8.2 \%$ \\
\hline
\end{tabular}

HER2-E, HER2-enriched; pCR, Pathological complete response; ER, Estrogen receptor; PR, Progesterone receptor; IHC, Immunohistochemistry

variables (Fig. 1c and d and Table 2; Additional file 1: Table S5). Among them, tumor size and nodal status before treatment were significantly associated with DRFS. Finally, high 5-year DRFS rates were observed as in the global population in patients with ROR-P low disease that did not achieve a pCR (5-year DRFS of $92.0 \%$ (95\% CI, 85.5-99.1\%) in all patients and of 97.4 \% (95 \% CI, 92.6$100.0 \%)$ in node-negative disease). No statistically significant interaction $(P=0.430)$ was observed between ROR-P (as a continuous variable) and PCR in DRFS analysis.
Intrinsic subtype association with chemotherapy response in all patients

The pCR rates across the intrinsic molecular subtypes were $6 \%, 16 \%, 37 \%$, and $38 \%$ for the Luminal A, Luminal B, HER2-enriched, and Basal-like subtypes, respectively. In a multivariable model, the intrinsic subtypes were independently associated with $\mathrm{pCR}$ after adjustment for age, tumor size, ER and PR statuses, histological grade, HER2 status, and study (Table 3 and Additional file 1: Table S6). Of note, ER and PR status 


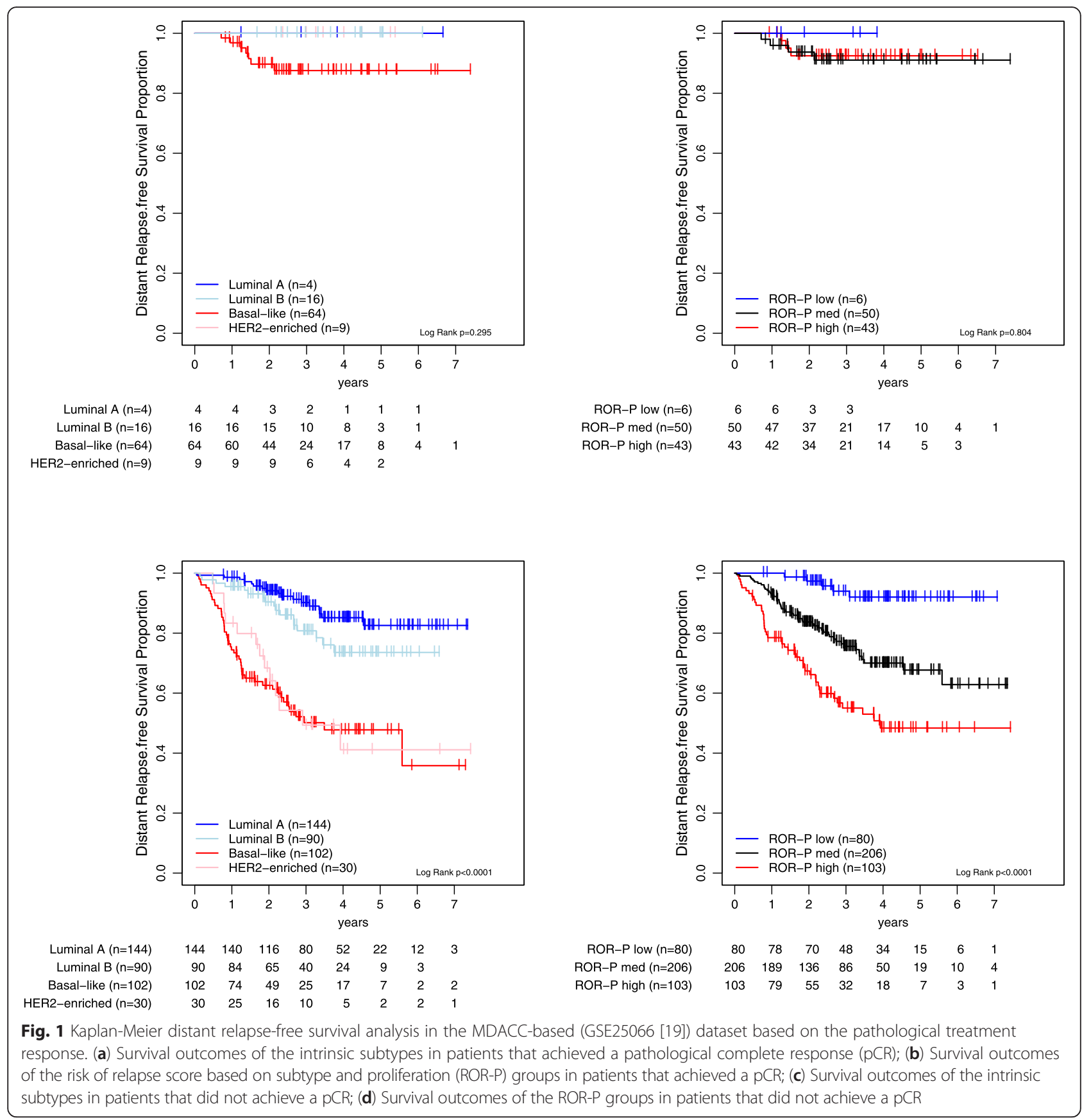

by immunohistochemistry (IHC) did not provide independent predictive information once intrinsic subtype was introduced into the model.

\section{pCR rates of the intrinsic subtypes across pathology-defined subgroups}

The intrinsic subtype classification was independently associated with $\mathrm{pCR}$ within $\mathrm{HR}^{+} / \mathrm{HER} 2^{-}, \mathrm{HER}^{+}$, and TNBC clinical subgroups (Table 4). Non-luminal (Basallike and HER2-enriched) tumors, as a group, showed higher $\mathrm{pCR}$ rates than luminal (Luminal $\mathrm{A}$ and $\mathrm{B}$ ) tumors in $\mathrm{HR}^{+} / \mathrm{HER} 2^{-}(30.0 \%$ vs. $8.9 \%$, adjusted $\mathrm{OR}=4.20$, 2.220-7.942), HER2 $2^{+}(45.8 \%$ vs. $14.3 \%$, adjusted OR = $5.22,1.478-18.460)$, and TNBC (38.5 \% vs. $18.5 \%$, adjusted $\mathrm{OR}=2.89,1.043-8.003$ ) diseases. Among the different subtypes, the Basal-like subtype showed consistent pCR rates above $35 \%$ across the three clinically-defined subgroups $\left(36 \%, 58 \%\right.$, and $37 \%$ in $\mathrm{HR}^{+} / \mathrm{HER}^{-}$, HER $2^{+}$, and TN subgroups, respectively). Finally, addition of the Claudin-low subtype to the PAM50 classification did not improve the ability to predict $\mathrm{pCR}$ in TNBC (Additional file 1: Table S7). 
Table 2 Cox model distant relapse-free survival (DRFS) analyses in patients with residual disease from the MDACC-based cohort (GSE25066 [19])

\begin{tabular}{|c|c|c|c|c|c|c|c|c|c|c|c|}
\hline \multirow[b]{2}{*}{ Variables } & \multirow[b]{2}{*}{$n$} & \multirow[b]{2}{*}{$\%$} & \multirow[b]{2}{*}{ 5-yr DRFS } & \multicolumn{4}{|c|}{ Univariate analysis } & \multicolumn{4}{|c|}{ Multivariable analysis } \\
\hline & & & & $\mathrm{HR}$ & Lower $95 \%$ & Upper $95 \%$ & $P$ value & $\overline{\mathrm{HR}}$ & Lower 95 \% & Upper $95 \%$ & $P$ value \\
\hline Age, years (cont. variable) & - & - & - & 1.0 & 0.98 & 1.01 & 0.590 & 0.98 & 0.97 & 1.01 & 0.129 \\
\hline \multicolumn{12}{|l|}{ Tumor size } \\
\hline T0-T2 & 216 & $56 \%$ & $74 \%$ & 1.0 & - & - & - & 1.0 & - & - & - \\
\hline T3-T4 & 173 & $44 \%$ & $61 \%$ & 2.1 & 1.38 & 3.07 & $<0.001$ & 1.5 & 1.16 & 1.92 & 0.002 \\
\hline \multicolumn{12}{|l|}{ Node status } \\
\hline No & 126 & $32 \%$ & $85 \%$ & 1.0 & - & - & - & 1.0 & - & - & - \\
\hline $\mathrm{N} 1-3$ & 263 & $68 \%$ & $66 \%$ & 3.3 & 1.90 & 5.71 & $<0.001$ & 2.9 & 1.62 & 5.37 & $<0.001$ \\
\hline \multicolumn{12}{|l|}{ ER IHC } \\
\hline Positive & 129 & $34 \%$ & $78 \%$ & 1.0 & - & - & - & 1.0 & - & - & - \\
\hline Negative & 255 & $66 \%$ & $47 \%$ & 4.0 & 2.69 & 6.02 & $<0.001$ & 1.8 & 0.87 & 3.56 & 0.114 \\
\hline \multicolumn{12}{|l|}{$P R \| C$} \\
\hline Positive & 175 & $46 \%$ & $79 \%$ & 1.0 & - & - & - & 1.0 & - & - & - \\
\hline Negative & 208 & $54 \%$ & $54 \%$ & 3.3 & 2.15 & 5.05 & $<0.001$ & 1.2 & 0.62 & 2.13 & 0.654 \\
\hline \multicolumn{12}{|l|}{ HER2 STATUS } \\
\hline Negative & 373 & $99 \%$ & $68 \%$ & 1.0 & - & - & - & 1.0 & - & - & - \\
\hline Positive & 3 & $1 \%$ & NA & 1.1 & 0.16 & 8.16 & 0.900 & 0.5 & 0.07 & 3.65 & 0.485 \\
\hline \multicolumn{12}{|l|}{ Histological grade } \\
\hline 1 & 28 & $8 \%$ & $96 \%$ & 1.0 & - & - & - & 1.0 & - & - & - \\
\hline 2 & 160 & $44 \%$ & $73 \%$ & 6.1 & 0.83 & 44.43 & 0.076 & 2.75 & 0.36 & 20.94 & 0.33 \\
\hline 3 & 175 & $48 \%$ & $60 \%$ & 10.9 & 1.51 & 78.73 & 0.018 & 2.54 & 0.33 & 19.66 & 0.37 \\
\hline \multicolumn{12}{|l|}{ PAM50 } \\
\hline Luminal A & 144 & $37 \%$ & $83 \%$ & 1.0 & - & - & - & 1.0 & - & - & - \\
\hline Luminal B & 90 & $23 \%$ & $74 \%$ & 1.8 & 0.90 & 3.46 & 0.097 & 1.4 & 0.69 & 2.82 & 0.360 \\
\hline HER2-E & 30 & $8 \%$ & $41 \%$ & 5.9 & 3.38 & 10.30 & $<0.001$ & 2.7 & 1.15 & 6.41 & 0.023 \\
\hline Basal-like & 102 & $26 \%$ & $48 \%$ & 5.3 & 2.63 & 10.84 & $<0.001$ & 2.8 & 1.19 & 6.42 & 0.018 \\
\hline Normal-like & 23 & $6 \%$ & $82 \%$ & 1.6 & 0.55 & 4.85 & 0.380 & - & - & - & - \\
\hline
\end{tabular}

HER2-E, HER2-enriched; pCR, Pathological complete response; ER, Estrogen receptor; PR, Progesterone receptor; IHC, Immunohistochemistry

\section{TNBCtype association with chemotherapy response in TNBC}

Of the 350 TNBCs, 60 (17.1\%) were identified by the TNBCtype online tool [26] as having high ESR1 levels (Fig. 2) and thus were removed from many of the subsequent analyses because they are not considered a "class" by the TNBCtype tool. The intrinsic subtype distribution within this ESR1-high TNBCtype group was: Basal-like ( $\mathrm{n}=20,33.3 \%)$, Normal-like $(\mathrm{n}=17,28.3 \%)$, Luminal A $(\mathrm{n}=14,23.3 \%)$, Luminal $\mathrm{B}(\mathrm{n}=5,8.3 \%)$, and HER2enriched $(n=4,6.7 \%)$. As predicted, the levels of ESR1 mRNA in the TNBCtype ESR1-high group were significantly higher than in the ESR1-low group; however, the levels of ESR1 mRNA in the ESR1-high group were significantly lower than in the group with clinically $\mathrm{ER}^{+}$ disease by IHC (Additional file 1: Figure S3).
The distribution of the PAM50 intrinsic subtypes within the TNBCtype subgroups was similar to previous reports where virtually all TNBCtype LAR tumors were non-Basal-like (i.e. HER2-enriched or luminal), and $42 \%$ of MSL tumors were Normal-like (Additional file 1: Table S8 and Figure S4-5). Of note, $12.1 \%$ of TNBCs subtyped by the TNBCtype (or $10.0 \%$ of all TNBCs) were identified as UNS, and $86.0 \%$ of these were of the Basal-like subtype by PAM50; thus, $27 \%$ of the 350 clinically defined TNBCs were not assigned a biological group (i.e. either ESR1-high or UNS) by the TNBCtype tool (Fig. 2).

Of the remaining 290 TNBC sample set (350 TNBC 60 removed for high ESR1), 271 patients with TNBC had response data (Additional file 1: Table S9). In this subset, the TNBCtype classification was not found to be 
Table 3 Logistic regression model analyses of chemotherapy response in the combined cohort ${ }^{\mathrm{a}}$

\begin{tabular}{|c|c|c|c|c|c|c|c|c|c|c|c|c|}
\hline \multirow[b]{2}{*}{ Signatures } & \multirow[b]{2}{*}{$n$} & \multirow[b]{2}{*}{ pCR rate } & \multicolumn{5}{|c|}{ Univariate analysis } & \multicolumn{5}{|c|}{ Multivariable analysis } \\
\hline & & & OR & Lower 95 \% & Upper $95 \%$ & $P$ value & auROC & OR & Lower 95 \% & Upper $95 \%$ & $P$ value & auROC \\
\hline Age, years (cont. variable) & - & - & 1.0 & 0.97 & 1.00 & 0.027 & 0.547 & 0.99 & 0.97 & 1.01 & 0.178 & 0.744 \\
\hline \multicolumn{13}{|l|}{ Tumor size } \\
\hline T0-T2 & 556 & $24 \%$ & 1.0 & - & - & - & \multirow[t]{2}{*}{0.519} & 1.0 & - & - & \multicolumn{2}{|l|}{-} \\
\hline T3-T4 & 353 & $12 \%$ & 0.8 & 0.55 & 1.02 & 0.071 & & 0.6 & 0.50 & 0.84 & \multicolumn{2}{|l|}{0.001} \\
\hline \multicolumn{13}{|l|}{ ER IHC } \\
\hline Positive & 487 & $11 \%$ & 1.0 & - & - & - & \multirow[t]{2}{*}{0.679} & 1.0 & - & - & \multicolumn{2}{|l|}{-} \\
\hline Negative & 415 & $37 \%$ & 4.7 & 3.39 & 6.61 & $<0.001$ & & 1.8 & 0.99 & 3.34 & \multicolumn{2}{|l|}{0.052} \\
\hline \multicolumn{13}{|l|}{$P R \| H C$} \\
\hline Positive & 393 & $12 \%$ & 1.0 & - & - & - & \multirow[t]{2}{*}{0.643} & 1.0 & - & - & \multicolumn{2}{|l|}{-} \\
\hline Negative & 507 & $33 \%$ & 3.8 & 2.64 & 5.36 & $<0.001$ & & 1.1 & 0.65 & 1.89 & \multicolumn{2}{|l|}{0.716} \\
\hline \multicolumn{13}{|l|}{ HER2 STATUS } \\
\hline Negative & 799 & $22 \%$ & 1.0 & - & - & - & \multirow[t]{2}{*}{0.533} & 1.0 & - & - & \multicolumn{2}{|l|}{-} \\
\hline Positive & 88 & $35 \%$ & 2.1 & 1.30 & 3.30 & 0.002 & & 1.3 & 0.64 & 2.51 & \multicolumn{2}{|l|}{0.492} \\
\hline \multicolumn{13}{|l|}{ PAM50 } \\
\hline Luminal A & 281 & $6 \%$ & 1.0 & - & - & - & \multirow[t]{5}{*}{0.719} & 1.0 & - & - & \multicolumn{2}{|l|}{-} \\
\hline Luminal B & 168 & $16 \%$ & 3.0 & 1.57 & 5.64 & 0.001 & & 3.3 & 1.72 & 6.45 & \multicolumn{2}{|l|}{$<0.001$} \\
\hline HER2-E & 93 & $37 \%$ & 8.9 & 4.69 & 17.09 & $<0.001$ & & 6.1 & 2.75 & 13.38 & \multicolumn{2}{|l|}{$<0.001$} \\
\hline Basal-like & 296 & $38 \%$ & 9.6 & 5.49 & 15.40 & $<0.001$ & & 6.1 & 2.94 & 12.66 & \multicolumn{2}{|l|}{$<0.001$} \\
\hline Normal-like & 72 & $29 \%$ & 6.4 & 3.16 & 12.96 & $<0.001$ & & - & - & - & \multicolumn{2}{|l|}{-} \\
\hline \multicolumn{13}{|l|}{ STUDY } \\
\hline HORAK & 253 & $27 \%$ & 1.0 & - & - & - & \multirow[t]{4}{*}{0.553} & 1.0 & - & - & - & \\
\hline ISPY & 54 & $31 \%$ & 1.2 & 0.65 & 2.32 & 0.532 & & 1.3 & 0.55 & 3.16 & 0.540 & \\
\hline MDACC508 & 488 & $20 \%$ & 0.7 & 0.48 & 0.97 & 0.032 & & 0.9 & 0.58 & 1.33 & 0.526 & \\
\hline MIYAKE & 115 & $23 \%$ & 0.8 & 0.49 & 1.37 & 0.443 & & 1.0 & 0.55 & 1.95 & 0.910 & \\
\hline
\end{tabular}

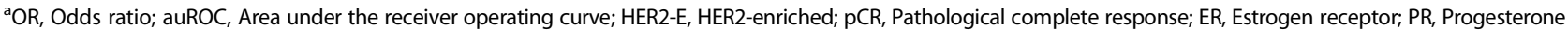
receptor; IHC, Immunohistochemistry

significantly associated with $\mathrm{pCR}$ in univariate $(P=$ $0.762)$ or multivariable analyses $(P=0.836)$. Of note, only eight patients had luminal $\mathrm{A} / \mathrm{B}$ disease and their $\mathrm{pCR}$ rate was $25 \%$ versus $41 \%$ in non-luminal (Basal-like and HER2-enriched combined) tumors $(\mathrm{OR}=0.477$, 0.094-2.410).

Finally, we explored the ability of the TNBCtype classification to predict $\mathrm{pCR}$ within TNBC if the ESR1-high samples were included as an eighth subtype (i.e. ESR1-high).
Interestingly, the $\mathrm{pCR}$ rate of the TNBCtype subtypes, as a single group, was significantly higher than the pCR rate of the 'excluded' TNBC ESR1-high group (39.9\% vs. $23.2 \%$, $\mathrm{OR}=2.970,1.221-7.222)$. In the entire TNBC population $(\mathrm{n}=350)$, the TNBCtype classification that included the ESR1-high group was found significantly associated with pCR in multivariable analysis $(P=0.020)$ but not in univariate analysis $(P=0.239)$. When the TNBCtype + ESR1high classification was included first in a multivariable

Table 4 Association of the intrinsic subtypes with chemotherapy response across the various pathology-based groups

\begin{tabular}{|c|c|c|c|c|c|c|c|c|c|c|c|}
\hline & \multicolumn{2}{|c|}{ All patients } & \multicolumn{2}{|c|}{ Luminal A } & \multicolumn{2}{|c|}{ Luminal B } & \multicolumn{2}{|c|}{ HER2-enriched } & \multicolumn{2}{|c|}{ Basal-like } & \multirow[t]{2}{*}{$P$ value* } \\
\hline & $\bar{n}$ & $\overline{p C R}$ & $\mathrm{n}$ & $\overline{\mathrm{pCR}}$ & $\mathrm{n}$ & $\overline{\mathrm{pCR}}$ & $\bar{n}$ & $\mathrm{pCR}$ & $\mathrm{n}$ & $\overline{p C R}$ & \\
\hline All subgroups & 838 & $23 \%$ & 281 & $6 \%$ & 168 & $16 \%$ & 93 & $37 \%$ & 296 & 38 \% & $<0.001$ \\
\hline $\mathrm{HR}^{+} / \mathrm{HER}^{-}$ & 451 & $12 \%$ & 239 & $5 \%$ & 143 & $15 \%$ & 25 & $16 \%$ & 44 & $36 \%$ & $<0.001$ \\
\hline HER2 ${ }^{+}$ & 76 & $34 \%$ & 16 & $0 \%$ & 12 & $33 \%$ & 36 & $42 \%$ & 12 & $58 \%$ & 0.011 \\
\hline $\mathrm{HR}^{-} / \mathrm{HER}^{-}$(TN) & 292 & $37 \%$ & 19 & $26 \%$ & 8 & $0 \%$ & 30 & $47 \%$ & 235 & $37 \%$ & 0.011 \\
\hline
\end{tabular}

*Likelihood ratio tests: adjusting clinical features: age, clinical stage, clinical nodal status and study cohort. Hormone receptors status and HER2 status were also included in "all subgroups"

pCR, Pathological complete response; ER, Estrogen receptor; PR, Progesterone receptor 


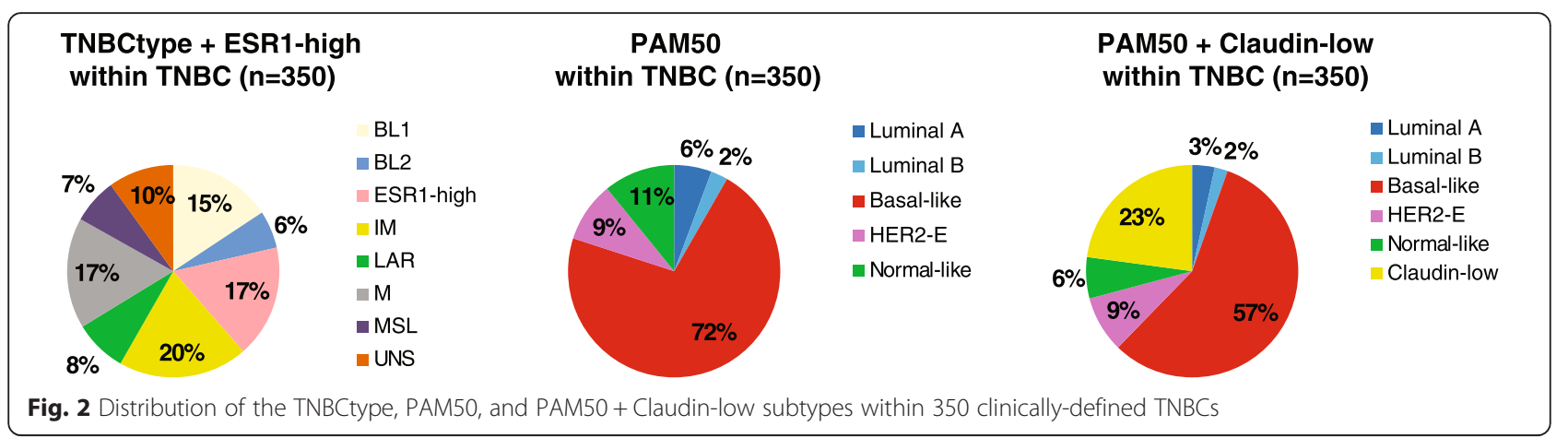

model, addition of the PAM50 classification did not add independent predictive information, but was trending toward significance $(P=0.096)$. Similar results were obtained if the PAM50 classification was included first into the multivariable model and the TNBCtype + ESR1-high classification was added second $(P=0.088)$.

\section{Training and testing gene expression-based models predictive of $\mathrm{pCR}$}

We explored the ability of newly derived gene expressionbased models to predict pCR in three different subgroups: all patients, patients with Basal-like disease, and patients with Luminal disease (Luminal A and B combined). To accomplish this, we built a model in the MDACC-based cohort (training dataset) and then tested the same model on the other cohorts (testing datasets) (Additional file 1: Figure S6-8).

In all patients, a gene expression-based model was identified in the MDACC-based cohort with an auROC of 0.80 $(P<0.0001)$. This model predicted $\mathrm{pCR}$ in each testing datasets with auROC between 0.67-0.75 $(P<0.001)$, and in the combined testing dataset (auROC 0.69, $P<0.0001$ ). The gene signatures that composed the model and whose high scores were associated with residual disease were correlation to the Luminal A centroid, correlation to PTEN present, and the Luminal A subtype (Additional file 1: Figure S6). Conversely, the gene signatures that composed the model and whose high scores were associated with pCR were correlation to the Basal-like centroid, correlation to PTEN absent [28], a beta-catenin signature, and a fetal mammary stem cell signature $[29,30]$.

In patients with Basal-like disease, a gene expressionbased model was identified in the MDACC-based cohort ( $\mathrm{n}=166$; auROC $=0.82, \quad P<0.0001)$. This model predicted pCR in Horak et al. [19] (auROC 0.63, $P=0.018$ ) and in the combined cohort of testing sets $(n=130$; auROC $0.62, P=0.011$ ). Gene signatures that composed the model and whose high score were associated with residual disease were related to stromal/fibroblast-related biological processes (Additional file 1: Figure S7). Conversely, gene signatures that composed the model and whose high scores were associated with $\mathrm{pCR}$ were associated with histone/chromatin remodeling.

Finally, in patients with Luminal disease, a gene expression-based model was identified in the MDACCbased cohort $(n=254$; auROC $=0.82, P<0.0001)$. This model predicted pCR in Miyake et al. [20] (auROC $0.76, P=0.03)$ and in the combined cohort of testing sets $(\mathrm{n}=195$; auROC $0.64, P=0.014)$. The only gene signature that composed the model and whose high score was associated with residual disease was correlation to TP53 wild-type status, whereas the only gene signature that composed the model and whose high score was associated with pCR was correlation to TP53 mutation (Additional file 1: Figure S8). Of note, both TP53 signatures composed our previously reported TP53 loss/mutation predictor [31].

\section{Discussion}

Herein, we evaluated the association of the intrinsic subtypes of breast cancer with response and survival outcomes in a large combined dataset of newly diagnosed patients treated with multi-agent neoadjuvant chemotherapy and we made the following observations. First, the intrinsic subtypes of breast cancer provided independent prognostic information beyond standard clinical-pathological variables. Second, within patients that do not achieve a $\mathrm{pCR}$, the ROR-P predictor can identify a group of patients with clinically node-negative disease with an excellent survival outcome at 5-years. Third, the intrinsic subtypes predict $\mathrm{pCR}$ and their predictive value is independent of standard clinicopathological variables. Fourth, the Basal-like subtype identifies a group of patients with a pCR rate $>35 \%$ across all pathologybased cohorts evaluated, including TNBC. Fifth, neither the identification of the Claudin-low subtype nor the recently reported seven-TNBC subtype classification predicted pCR within the large TNBC data set tested here, whereas the Luminal versus non-Luminal separation did predict pCR. Sixth, robust gene expression-based models predictive of $\mathrm{pCR}$ can be identified within all 
patients, Basal-like disease, and Luminal disease; however, additional validation of these new predictors is needed.

The intrinsic subtypes have previously been associated with outcome in patients that have not received adjuvant systemic therapy [32] and in patients that have received adjuvant endocrine therapy-only [33-38]. More recently, similar data has been observed in patients that have received adjuvant multi-agent chemotherapy, including CMF, anthracycline-based, and anthracycline/taxanebased chemotherapy regimens [5, 8, 33]. Concordant with the results of these studies, we observed an independent association of the intrinsic subtypes with DRFS in a population treated with cytotoxic and endocrine therapy (if $\mathrm{HR}^{+}$). Interestingly, this association with outcome was observed despite the fact that $20.3 \%$ of the patients in the Hatzis et al. [19] dataset had an outstanding survival outcome at 5-years after achieving a pCR. This data reaffirms the strong prognostic ability of intrinsic subtyping in the context of standard adjuvant therapy.

The prognostic abilities of the PAM50 ROR-P have been clinically validated in two large retrospective cohorts from the ABCSG08 and transATAC phase III trials, where patients with surgically removed tumors received adjuvant endocrine therapy only $[36,37]$. In this context, patients with a low ROR-P score have an outcome of distant metastasis-free survival at 10-years of $97.5 \%$ [32], and these patients might be safely spared adjuvant (or neoadjuvant) chemotherapy. In our cohort of patients treated with neoadjuvant cytotoxic and adjuvant endocrine therapy (if $\mathrm{HR}^{+}$), ROR-P at diagnosis independently predicted DRFS and identified a low risk group of patients, especially within clinically node-negative disease, with an outstanding outcome (DRFS $>95 \%$ at 5years). Similar results have been obtained with other prognostic signatures tested in patients with early breast cancer treated with and without multi-agent chemotherapy [39]. These nearly identical DRFS survival times with or without chemotherapy suggest that the potential survival benefit from neoadjuvant chemotherapy in patients with newly diagnosed breast cancer that is clinically node-negative and ROR-P low might be rather small, if any. In Hatzis et al. [19], the proportion of patients with ROR-P low within clinically node-negative disease was $26.8 \%$. If the main objective of neoadjuvant chemotherapy is to increase survival, then these patients with an outstanding baseline prognosis should be spared the toxic side-effects of chemotherapy and undergo surgical removal of their tumors.

Molecular classification of TNBC into subgroups that might be therapeutically relevant is an area of active and ongoing research. For example, the PAM50 assay identifies all the intrinsic molecular subtypes within TNBC, although Basal-like disease predominates [40]. In addition, we have identified and characterized a rare but relevant intrinsic subtype known as Claudin-low [4]. Interestingly, the intrinsic subtypes within TNBC share the same molecular features as the same subtypes within non-TNBC with the exception of the TNBC HER2-enriched tumors that do not show amplification of the ERBB2 17q amplicon $[5,41]$. In our combined cohort of 350 TNBC cases, intrinsic subtyping, and especially the luminal versus non-luminal distinction, was found to be associated with pCR following neoadjuvant chemotherapy. However, the addition of the Claudin-low classification to the PAM50 classification did not improve these $\mathrm{pCR}$ versus no $\mathrm{pCR}$ predictions.

In addition, Lehmann et al. [17] have classified TNBC into seven subtypes (BL1, BL2, Immunomodulatory, LAR, Mesenchymal, MSL and UNS). This seven-subtype classification of TNBC has been found to be associated with pCR in an independent cohort of 143 patients with TNBC treated with anthracycline/taxane-based chemotherapy [18]. In our combined cohort of 290 TNBC cases with seven-subtype information, the Lehmann et al. [17] classification was not found to be significantly associated with pCR. However, concordant with a previous report, BL1 showed the highest $\mathrm{pCR}$ rate (i.e. $47 \%$ ) and BL2 the lowest pCR rate (i.e. $28 \%$ ). Surprisingly, the LAR group, which was found to have a $10 \%(2 / 20)$ pCR rate in a previous report [18], showed a $37 \%$ pCR rate in this larger combined cohort. This difference might be due to the fact that $71.4 \%(20 / 28)$ of LAR tumors in our combined cohort were of the HER2-enriched subtype, a group of tumors highly responsive to chemotherapy, and only $17.9 \%(5 / 28)$ were of the Luminal A/B subtype.

Two important issues of the Lehmann et al. [17] classification need to be taken into account. First, this classification ignores the Normal-like/normal tissue distinction. In other words, triple-negative tumors that are highly contaminated with normal breast tissue, which represent $11-16 \%$ of the samples found in publicly available microarray datasets [17], are now classified into "tumor" subtypes. Whereas PAM50 identifies these tumors as being more similar to true normal breast samples (i.e. Normallike) than to any tumor subtype, the Lehmann et al. [17] classification calls them as if they were a tumor (mostly MSL), although the Normal-like samples can also be observed in other subtype categories [40, 42]. Second, a substantial proportion of TNBC samples ( 13-16\%) coming from the Lehmann et al. [17] classification were either not considered to be TNBC by gene expression and are removed (i.e. ESR1-high), or they fall into the unclassified or unstable (UNS) group, which is composed of a mix of tumors that only share the feature that they cannot be classified into one of the other six tumor subtypes.

This study also has other limitations that need to be highlighted. First, this was a retrospective and exploratory analysis of four datasets of patients treated with 
multi-agent chemotherapy; thus, we did not test a prespecified hypothesis. Second, we used the research-based version of the PAM50 assay and not the standardized version that is currently commercially available. Third, we could not evaluate the predictive ability of the intrinsic subtypes to specific regimens or schedules. Fourth, we used the pathological data as provided in each publication and different definitions and cutoffs might have been used to determine the positivity of each biomarker. Thus, the results might have differed if ER, PR, and HER2 status had been centrally confirmed. Nonetheless, we and others have reported that, even within centrally confirmed TNBC, all the intrinsic molecular subtypes can be identified [15]. Fifth, Ki-67 by IHC was not available in any of the four datasets and thus we could not explore the ability of this biomarker to predict $\mathrm{pCR}$ following chemotherapy or survival outcome in the presence of the intrinsic subtypes or histological grade [43], especially within $\mathrm{HR}^{+} / \mathrm{HER}^{-}$disease. Sixth, the survival outcomes were only available in one of the datasets evaluated. Finally, the cutoffs to define the three risk groups of ROR-P were based on a large node-negative cohort that did not receive adjuvant systemic therapy [24]. These cutoffs might differ from the current standardized PAM50 version that takes into account tumor size and that defines the low risk group as those patients with a risk of distant relapse at 10 -years below $3 \%[36,37]$.

\section{Conclusion}

To conclude, intrinsic subtyping at diagnosis provides useful prognostic and predictive information for neoadjuvant chemotherapy-treated patients. The absolute benefit of chemotherapy in early breast cancer with clinically nodenegative disease might be low if predicted to be ROR-P low risk at diagnosis. Further studies are needed to determine the role of intrinsic subtyping in treatment decisionmaking at diagnosis of breast cancer.

\section{Availability of data and materials}

Four clinically annotated microarray-based breast cancer datasets were evaluated from the public domain (GSE25066 [19], GSE32646 [20], GSE41998 [21] and GSE22226 [22]). The sample names and subtype calls can be found in Additional file 2: Supplemental Data.

\section{Additional files}

Additional file 1: Table S1. Cox model DRFS analyses including intrinsic subtype in all patients from the MDACC-based cohort (GSE25066). Table S2. Cox model DRFS analyses including ROR-P in all patients from the MDACC-based cohort (GSE25066). Table S3. Cox model DRFS analyses including intrinsic subtype in patients that achieved a pCR from the MDACC-based cohort (GSE25066). Table S4. Cox model DRFS analyses including ROR-P in patients that achieved a pCR from the MDACC-based cohort (GSE25066). Table S5. Cox model DRFS analyses including ROR-P in patients with residual disease from the MDACC-based cohort (GSE25066). Table S6. Distribution of the PAM50 subtypes within the TNBCtype groups and vice versa. Table S7. Association of the TNBCtype subtypes with chemotherapy response in triple-negative breast cancer. Figure S1. CONSORT diagram of the various cohorts evaluated in this study. Figure S2. Kaplan-Meier distant relapse-free survival analysis in MDACC-based (GSE25066 [13]) dataset set. (A) Survival outcomes of the ROR-P groups in all patients. (B) Survival outcomes of the ROR-P groups in patients with clinically nodenegative disease. Figure S3. Levels of ESR1 across TNBCtype ESR1-low group, TNBCtype ESR1-high group and $\mathrm{ER}^{+}$group. Median expression of ESR1 in the PAM50 training dataset reported in Parker et al. [24] has been set to zero. Figure S4. Distribution of the TNBCtype subtypes and ESR1-high group within the PAM50 subtypes in TNBC. Figure S5. Distribution of the TNBCtype subtypes and ESR1-high group within the PAM50 + Claudinlow subtypes in TNBC. Figure S6. Training and testing gene expression-based models predictive of pCR in all patients. Figure S7. Training and testing gene expression-based models predictive of $\mathrm{pCR}$ in patients with Basal-like disease. Figure S8. Training and testing gene expression-based models predictive of pCR in patients with luminal (A/B) disease. (DOCX $819 \mathrm{~kb})$

Additional file 2: Supplemental data. (XLSX 897 kb)

\section{Abbreviations}

auROC: Area under the receiver operating characteristic; BL1: Basal 1; BL2: Basal 2; DRFS: Distant relapse-free survival; ER: Estrogen receptor; ESR1: ER gene; IHC: Immunohistochemistry; LAR: Luminal androgen receptor; MSL: Mesenchymal stem cell; pCR: Pathological complete response; PR: Progesterone receptor; ROR-P: Risk of relapse score based on subtype and proliferation; TNBC: Triple-negative breast cancer; UNS: Unstable.

\section{Competing interests}

CMP is an equity stock holder of BioClassifier LLC and University Genomics, and has filed a patent on the PAM50 assay. Uncompensated advisory role of AP for Nanostring Technologies. The other authors declare that they have no competing interests.

\section{Authors' contributions}

Study conception and design: AP and CMP. Acquisition of data: all authors. Analysis and interpretation of data: all authors. All authors were involved in drafting the article or revising it critically for important intellectual content, and approved the final version of the manuscript.

\section{Acknowledgements}

This work was supported by funds from the NCI Breast SPORE program (P50-CA58223-09A1), by RO1-CA148761 (CMP), by Instituto de Salud Carlos III - PI13/01718 (AP), by a Career Catalyst Grant from the Susan Komen Foundation (AP), by Banco Bilbao Vizcaya Argentaria (BBVA) Foundation (AP) and by the Breast Cancer Research Foundation. The funders did not have any role in the study design, conduct, or decision to submit the manuscript for publication.

\section{Author details}

${ }^{1}$ Translational Genomics Group, Vall d'Hebron Institute of Oncology (VHIO), Barcelona, Spain. ${ }^{2}$ Translational Genomics and Targeted Therapeutics in Solid Tumors, August Pi i Sunyer Biomedical Research Institute (IDIBAPS), Rosselló, 149, 08036 Barcelona, Spain. ${ }^{3}$ Department of Medical Oncology, Hospital Clínic de Barcelona, Barcelona, Spain. ${ }^{4}$ Lineberger Comprehensive Cancer Center, University of North Carolina, Chapel Hill, NC, USA. ${ }^{5}$ Clinical Trials \& Statistics Unit, The Institute of Cancer Research, Belmont, UK. ${ }^{6}$ Department of Genetics, University of North Carolina, Chapel Hill, NC, USA. ${ }^{7}$ Department of Pathology \& Laboratory Medicine, University of North Carolina, Chapel Hill, NC, USA.

Received: 7 September 2015 Accepted: 30 November 2015

Published online: 18 December 2015

\section{References}

1. Prat A, Ellis MJ, Perou CM. Practical implications of gene-expression-based assays for breast oncologists. Nat Rev Clin Oncol. 2012;9:48-57. 
2. Prat A, Galván P, Jimenez B, Buckingham W, Jeiranian HA, Schaper C, et al. Prediction of response to neoadjuvant chemotherapy using core needle biopsy samples with the prosigna assay. Clin Cancer Res 2015. In Press.

3. Sorlie T, Perou C, Tibshirani R, Aas T, Geisler S, Johnsen H, et al. Gene expression patterns of breast carcinomas distinguish tumor subclasses with clinical implications. Proc Natl Acad Sci U S A. 2001;98:10869-74.

4. Prat A, Parker J, Karginova O, Fan C, Livasy C, Herschkowitz Jl, et al. Phenotypic and molecular characterization of the claudin-low intrinsic subtype of breast cancer. Breast Cancer Res. 2010;12:R68.

5. Martín M, Prat A, Rodríguez-Lescure Á, Caballero R, Ebbert MT, Munárriz B, et al. PAM50 proliferation score as a predictor of weekly paclitaxel benefit in breast cancer. Breast Cancer Res Treat. 2013;138:457-66.

6. Caan BJ, Sweeney C, Habel LA, Kwan ML, Kroenke CH, Weltzien EK, et al. Intrinsic subtypes from the PAM50 gene expression assay in a population-based breast cancer survivor cohort: prognostication of short- and long-term outcomes. Cancer Epidemiol Biomarkers Prev. 2014;23:725-34.

7. Chia SK, Bramwell VH, Tu D, Shepherd LE, Jiang S, Vickery T, et al. A 50-gene intrinsic subtype classifier for prognosis and prediction of benefit from adjuvant tamoxifen. Clin Cancer Res. 2012;18:4465-72.

8. Cheang MCU, Voduc KD, Tu D, Jiang S, Leung S, Chia SK, et al. Responsiveness of intrinsic subtypes to adjuvant anthracycline substitution in the NCIC.CTG MA.5 randomized trial. Clin Cancer Res. 2012;18:2402-12.

9. Prat A, Pineda E, Adamo B, Galván P, Fernández A, Gaba L, et al. Clinical implications of the intrinsic molecular subtypes of breast cancer. Breast. 2015;24 Suppl 2:S26-35.

10. Perou CM, Sorlie T, Eisen MB, van de Rijn M, Jeffrey SS, Rees CA, et al. Molecular portraits of human breast tumours. Nature. 2000;406:747-52.

11. Prat A, Perou CM. Deconstructing the molecular portraits of breast cancer. Mol Oncol. 2011;5:5-23.

12. Prat A, Bianchini G, Thomas M, Belousov A, Cheang MC, Koehler A, et al. Research-based PAM50 subtype predictor identifies higher responses and improved survival outcomes in HER2-positive breast cancer in the NOAH study. Clin Cancer Res. 2014;20:511-21.

13. Cheang MCU, Martin M, Nielsen TO, Prat A, Voduc D, Rodriguez-Lescure A, et al. Defining breast cancer intrinsic subtypes by quantitative receptor expression. Oncologist. 2015;20:474-82.

14. Prat A, Cheang MCU, Martín M, Parker JS, Carrasco E, Caballero R, et al. Prognostic significance of progesterone receptor-positive tumor cells within immunohistochemically defined luminal A breast cancer. J Clin Oncol. 2013;31:203-9.

15. Prat A, Lluch A, Albanell J, Barry WT, Fan C, Chacón Jl, et al. Predicting response and survival in chemotherapy-treated triple-negative breast cancer. Br J Cancer. 2014;111(8):1532-41

16. Tutt A, Ellis P, Kilburn L, Gilett G, Pinder S, Abraham J, et al. The TNT trial: A randomized phase III trial of carboplatin (C) compared with docetaxel (D) for patients with metastatic or recurrent locally advanced triple negative or BRCA1/2 breast cancer (CRUK/07/012). San Antonio Breast Cancer Symposium. 2014;2012:S3-01.

17. Lehmann BD, Bauer JA, Chen X, Sanders ME, Chakravarthy AB, Shyr Y, et al. Identification of human triple-negative breast cancer subtypes and preclinical models for selection of targeted therapies. J Clin Invest. 2011;121:2750-67.

18. Masuda H, Baggerly KA, Wang Y, Zhang Y, Gonzalez-Angulo AM, Meric-Bernstam F, et al. Differential response to neoadjuvant chemotherapy among 7 triple-negative breast cancer molecular subtypes. Clin Cancer Res. 2013;19:5533-40.

19. Hatzis C, Pusztai L, Valero V, Booser DJ, Esserman L, Lluch A, et al. A genomic predictor of response and survival following taxane-anthracycline chemotherapy for invasive breast cancer. JAMA. 2011;305:1873-81.

20. Miyake T, Nakayama T, Naoi Y, Yamamoto N, Otani Y, Kim SJ, et al. GSTP1 expression predicts poor pathological complete response to neoadjuvant chemotherapy in ER-negative breast cancer. Cancer Sci. 2012;103:913-20.

21. Horak CE, Pusztai L, Xing G, Trifan OC, Saura C, Tseng LM, et al. Biomarker analysis of neoadjuvant doxorubicin/cyclophosphamide followed by ixabepilone or paclitaxel in early-stage breast cancer. Clin Cancer Res. 2013;19:1587-95.

22. Esserman L, Berry D, Cheang MU, Yau C, Perou CM, Carey L, et al. Chemotherapy response and recurrence-free survival in neoadjuvant breast cancer depends on biomarker profiles: results from the I-SPY 1 TRIAL (CALGB 150007/150012; ACRIN 6657). Breast Cancer Res Treat. 2012;132:1049-62.

23. Nielsen TO, Parker JS, Leung S, Voduc D, Ebbert M, Vickery T, et al. A comparison of PAM50 intrinsic subtyping with immunohistochemistry and clinical prognostic factors in tamoxifen-treated estrogen receptor-positive breast cancer. Clin Cancer Res. 2010;16:5222-32.
24. Parker JS, Mullins M, Cheang MCU, Leung S, Voduc D, Vickery T, et al. Supervised risk predictor of breast cancer based on intrinsic subtypes. J Clin Oncol. 2009;27:1160-7.

25. Usary J, Zhao W, Darr D, Roberts PJ, Liu M, Balletta L, et al. Predicting drug responsiveness in human cancers using genetically engineered mice. Clin Cancer Res. 2013;19:4889-99.

26. Chen X, Li J, Gray WH, Lehmann BD, Bauer JA, Shyr Y, et al. TNBCtype: a subtyping tool for triple-negative breast cancer. Canc Informat. 2012;11:147-56.

27. Dennis Jr G, Sherman BT, Hosack DA, Yang J, Gao W, Lane HC, et al. DAVID: database for annotation, visualization, and Integrated discovery. Genome Biol. 2003:4:R60

28. Saal LH, Johansson P, Holm K, Gruvberger-Saal SK, She QB, Maurer M, et al. Poor prognosis in carcinoma is associated with a gene expression signature of aberrant PTEN tumor suppressor pathway activity. Proc Natl Acad Sci U S A. 2007;104:7564-9.

29. Spike BT, Engle DD, Lin JC, Cheung SK, La J, Wahl GM. A mammary stem cell population identified and characterized in late embryogenesis reveals similarities to human breast cancer. Cell Stem Cell. 2012;10:183-97.

30. Pfefferle A, Spike B, Wahl G, Perou CM. Luminal progenitor and fetal mammary stem cell expression features predict breast tumor response to neoadjuvant chemotherapy. Breast Cancer Res Treat. 2015;149:425-37.

31. Troester M, Herschkowitz J, Oh D, He X, Hoadley KA, Barbier CS, et al. Gene expression patterns associated with p53 status in breast cancer. BMC Cancer. 2006;6:276.

32. Fan C, Prat A, Parker J, Liu Y, Carey LA, Troester MA, et al. Building prognostic models for breast cancer patients using clinical variables and hundreds of gene expression signatures. BMC Med Genomics. 2011;4:3.

33. Bastien RR, Rodriguez-Lescure A, Ebbert MT, Prat A, Munáriz B, Rowe L, et al. PAM50 breast cancer subtyping by RT-qPCR and concordance with standard clinical molecular markers. BMC Med Genomics. 2012;5:44.

34. Prat A, Parker J, Fan C, Perou CM. PAM50 assay and the three-gene model for identifying the major and clinically relevant molecular subtypes of breast cancer. Breast Cancer Res Treat. 2012;135:301-6.

35. Prat A, Parker JS, Fan C, Cheang MC, Miller LD, Bergh J, et al. Concordance among gene expression-based predictors for ER-positive breast cancer treated with adjuvant tamoxifen. Ann Oncol. 2012;23(11):2866-73.

36. Dowsett M, Sestak I, Lopez-Knowles E, Sidhu K, Dunbier AK, Cowens JW, et al. Comparison of PAM50 risk of recurrence score with oncotype DX and IHC4 for predicting risk of distant recurrence after endocrine therapy. J Clin Oncol. 2013; 31:2783-90.

37. Filipits M, Nielsen TO, Rudas M, Greil R, Stöger H, Jakesz R, et al. The PAM50 risk-of-recurrence score predicts risk for late distant recurrence after endocrine therapy in postmenopausal women with endocrine-responsive early breast cancer. Clin Cancer Res. 2014;20:1298-305.

38. Gnant M, Filipits M, Greil R, Stoeger H, Rudas M, Bago-Horvath Z, et al. Predicting distant recurrence in receptor-positive breast cancer patients with limited clinicopathological risk: using the PAM50 Risk of Recurrence score in 1478 postmenopausal patients of the ABCSG-8 trial treated with adjuvant endocrine therapy alone. Ann Oncol. 2014;25:339-45.

39. Callari M, Cappelletti V, D'Aiuto F, Musella V, Lembo A, Petel F, et al. Subtype specific metagene-based prediction of outcome after neoadjuvant and adjuvant treatment in breast cancer. Clinical Cancer Research 2015. [Epub ahead of print].

40. Prat A, Adamo B, Cheang MCU, Anders CK, Carey LA, Perou CM. Molecular characterization of basal-like and non-basal-like triple-negative breast cancer. Oncologist. 2013;18:123-33.

41. Prat A, Carey LA, Adamo B, Vidal M, Tabernero J, Cortés J, et al. Molecular features and survival outcomes of the intrinsic subtypes within HER2positive breast cancer. J Natl Cancer Inst. 2014;106(8).pii: dju152. doi:10. 1093/jnci/dju152.

42. Mayer IA, Abramson VG, Lehmann BD, Pietenpol JA. New strategies for triple-negative breast cancer - deciphering the heterogeneity. Clin Cancer Res. 2014;20:782-90.

43. Lips EH, Mulder L, de Ronde JJ, Mandjes IA, Koolen BB, Wessels LF, et al. Breast cancer subtyping by immunohistochemistry and histological grade outperforms breast cancer intrinsic subtypes in predicting neoadjuvant chemotherapy response. Breast Cancer Res Treat. 2013;140:63-71. 